\title{
Otimização de parâmetros operacionais do pré-tratamento da palha de cana-de-açúcar
}

Operational parameters optimization of sugarcane straw pretreatment

\author{
M. S. R. Santos-Rocha ${ }^{1 *}$; B. Pratto $;$ R. M. R. G. Almeida ${ }^{2,3}$; A. J. G. Cruz ${ }^{1,4}$ \\ ${ }^{1}$ Programa de Pós-Graduação em Engenharia Química,Universidade Federal de São Carlos, 13565-905, São Carlos \\ $-S P$, Brasil \\ ${ }_{2}^{2}$ Programa de Pós-Graduação em Engenharia Química,Universidade Federal de Alagoas, 57072-970, Maceió-AL, \\ Brasil \\ ${ }^{3}$ Centro de Tecnologia,Universidade Federal de Alagoas, 57072-970, Maceió-AL, Brasil \\ ${ }^{4}$ Departamento de Engenharia Química, Universidade Federal de São Carlos, 13565-905, São Carlos - SP, Brasil \\ *santosmartha2014@gmail.com \\ (Recebido em 19 de fevereiro de 2017; aceito em de 31 de março de 2017)
}

\begin{abstract}
O compromisso de atender as necessidades do presente sem comprometer a possibilidade de suprir as necessidades das gerações futuras, torna a diversificação da matriz energética brasileira e mundial fator cada vez mais compulsório. Diante disso, o uso de biomassas lignocelulósicas para obtenção de combustíveis mais limpos do ponto de vista ambiental é amplamente discutido na atualidade. O etanol combustível pode ser obtido a partir de fontes alternativas de energia, como as biomassas lignocelulósicas. Esse tipo de matéria-prima possui elevado teor de açúcares fermentescíveis em sua composição. Porém, esses não se encontram diretamente disponíveis, sendo necessárias etapas adicionais para sua obtenção. Este trabalho teve como objetivo a otimização da etapa de pré-tratamento da palha de cana-de-açúcar, por meio de um delineamento experimental $2^{2}$ com três repetições no ponto central. As variáveis de estudo foram: a razão palha/água $(\mathrm{m} / \mathrm{v})$ na etapa de pré-tratamento hidrotérmico e a temperatura reacional do pré-tratamento alcalino sequencial ao hidrotérmico. $\mathrm{O}$ estudo mostrou que a deslignificação não promoveu melhora significativa na conversão da celulose em glicose na hidrólise enzimática, o que não justifica a realização dessa etapa adicional, nas condições avaliadas. Já o prétratamento hidrotérmico apresentou bons resultados, alcançando na etapa de hidrólise conversões superiores a $85 \%$, em especial para as razões palha/água 1:6,7 e 1:20 (m/v).
\end{abstract}

Palavras-chave: palha de cana-de-açúcar, otimização, pré-tratamento sequencial, pré-tratamento hidrotérmico, bioetanol

The commitment to meeting the needs of the present without compromising the ability to meet the needs of future generations makes the diversification of the Brazilian and the global energy matrix a compulsory factor. Thus, the use of lignocellulosic biomass to obtain cleaner fuels from an environmental point of view is widely discussed today. Fuel ethanol has been obtained from alternative energy sources, as lignocellulosic biomass. This type of biomass has high content of fermentable sugars. However, these sugars were not directly available, which need to include additional steps in the process to achieve this goal. This study aims to carry out an operational parameters optimization in the sugarcane straw pretreatment through a $2^{2}$ experimental design with three replications at the central point. The study variables were: straw-water ratio $(\mathrm{m} / \mathrm{v})$ in the hydrothermal pretreatment and the temperature reaction in the alkaline pretreatment, sequential to hydrothermal. The study reveals that the delignification did not promote significant improvements in glucose conversion during the enzymatic hydrolysis. Thus, this addition step was not justified, under the evaluated conditions. Furthermore, the hydrothermal pretreatment showed good results reaching conversions higher than $85 \%$ in the hydrolysis step, in particular for the straw-water ratios 1:6.7 and 1:20 (m/v).

Keywords: sugarcane straw, optimization, sequential pretreatment, hydrothermal pretreatment, bioethanol

\section{INTRODUÇÃO}

A busca pela diversificação da matriz energética mundial vem sendo motivada pela elevada demanda por combustíveis, pela instabilidade nos preços do petróleo e por restrições ambientais 
relacionadas ao uso de combustíveis fósseis. Diferentes fontes de energia renováveis estão sendo estudadas com o objetivo de complementar àquelas derivadas do petróleo. Nesse contexto, as biomassas lignocelulósicas surgem como uma boa alternativa para essa finalidade $[1,2,3]$.

Em relação ao uso de biomassas, o Brasil encontra-se em posição de destaque, visto que se localiza em uma região tropical, recebendo intensa radiação solar durante todo o ano. As condições climáticas existentes no país são, portanto, bastante favoráveis para a obtenção de energia a partir das biomassas [4]. Outro fator que corrobora essa afirmação é o fato do Brasil ter sido pioneiro na produção de etanol a partir da cana-de-açúcar (denominado de etanol de primeira geração, E1G). O país é o maior produtor mundial de etanol a partir dessa matériaprima [5, 6]. As biomassas lignocelulósicas, compostas principalmente por celulose, hemicelulose e lignina, apresentam grande potencial para produção de etanol. Após a obtenção dos carboidratos, presentes na celulose e hemicelulose, estes são convertidos por rota bioquímica a etanol. Exemplos de biomassas lignocelulósicas são a palha e o bagaço de canade-açúcar, o sabugo e a palha de milho e resíduos da indústria de papel e madeira. A palha de cana-de-açúcar é um dos mais importantes e abundantes resíduos do processamento da cana. Normalmente é deixada no campo como recobrimento do solo ou, em algumas situações, transportada para a usina para ser queimada nas caldeiras para obtenção de energia. A palha de cana-de-açúcar apresenta em sua composição entre 34-43\% de celulose, $23-38 \%$ de hemicelulose (majoritariamente xilanas), $11-25 \%$ de lignina e pequenas quantidades de ceras e compostos fenólicos de baixo peso molecular, denominados extrativos, além de componentes inorgânicos [7, 8, 9].

A grande diferença do processo de obtenção de etanol diretamente do caldo extraído da cana-de-açúcar (E1G), e razão pela qual a tecnologia de produção de etanol a partir de biomassas, denominado de etanol de segunda geração (E2G), ainda se apresenta desafiadora é o fato de que para converter os polissacarídeos a etanol necessita-se de duas etapas adicionais no processo: pré-tratamento e hidrólise da biomassa. A etapa de pré-tratamento é necessária para promover a desorganização do complexo lignocelulósico e reduzir a recalcitrância da celulose. A etapa de hidrólise é responsável pela obtenção dos açúcares (monômeros), que não se encontram diretamente disponível, como no caso das matérias sacarídeas $[6,10]$.

Muitos estudos têm apontado o pré-tratamento hidrotérmico como uma alternativa promissora, por ser uma tecnologia ambientalmente limpa. Esse tipo de pré-tratamento utiliza água quente sob alta pressão (1-3,5 MPa) e, nesse caso, a água atua como o solvente e deve estar entre 150 e $230{ }^{\circ} \mathrm{C}$. O pré-tratamento, portanto, é visto como promissor, pois a princípio não emprega reagentes químicos e não gera nenhum tipo de corrosão ao equipamento utilizado, reduzindo os custos operacionais e fixos $[11,12,13,14,15]$.

Um tratamento químico que pode ser realizado posteriormente ao hidrotérmico é o prétratamento com peróxido de hidrogênio alcalino, que é realizado em condições amenas de temperatura e pressão onde o peróxido se decompõe em oxigênio e água, sendo considerado de baixo impacto ambiental. Esse pré-tratamento adicional tem como objetivo principal promover a deslignificação da biomassa [16].

A etapa de hidrólise enzimática representa a maior dificuldade em tornar o etanol de segunda geração um processo industrialmente viável, devido a gargalos técnicos e econômicos $[17,18]$. Porém, perspectivas positivas são apresentadas por Viikari et al. (2012) [19], no qual os autores relataram que os custos associados a etapa de hidrólise enzimática tendem a diminuir significativamente nos próximos anos.

Oliveira et al. (2014) [7] avaliaram um processo combinado (pré-tratamento hidrotérmico seguido de deslignificação alcalina e posterior hidrólise enzimática) para a produção de bioetanol a partir da palha de cana-de-açúcar. O tratamento hidrotérmico foi conduzido entre 180 e $195{ }^{\circ} \mathrm{C}$, com tempo reacional entre 0 e 15 minutos. A deslignificação alcalina foi realizada com hidróxido de sódio e a maior solubilização de hemicelulose, bem como a maior conversão de celulose na hidrólise, foram alcançadas na condição de $190{ }^{\circ} \mathrm{C}$ e 10 minutos. Ademais, a etapa de deslignificação não promoveu melhora significativa na conversão da celulose a glicose na hidrólise enzimática. 
Santos et al. (2014) [3] realizaram tratamento hidrotérmico da palha de cana, utilizando planejamento experimental (variáveis: temperatura entre 170 e $210{ }^{\circ} \mathrm{C}$, tempo entre 5 e 15 minutos, e concentração palha/água entre 10 e 15\%). A melhor condição encontrada no tratamento hidrotérmico foi a $190{ }^{\circ} \mathrm{C}, 18$ minutos e $12,5 \%$ de relação palha/água.

Diante do exposto, esse trabalho teve como objetivo a otimização da etapa de pré-tratamento da palha de cana-de-açúcar por meio de um delineamento experimental $2^{2}$ com três repetições no ponto central.

\section{MATERIAL E MÉTODOS}

\subsection{MATERIAL}

A palha de cana-de-açúcar (RB 86-7515) utilizada neste trabalho foi gentilmente cedida pela Usina Ipiranga (Descalvado, SP). O extrato enzimático Cellic ${ }^{\circledR} \mathrm{Ctec} 2$ foi doado pela empresa Novozymes Latin America (Araucária, PR). Os demais reagentes de laboratório foram adquiridos em grau analítico e não sofreram qualquer tratamento antes de sua utilização.

\subsection{MÉTODOS}

\subsubsection{PRÉ-TRATAMENTO HIDROTÉRMICO}

O pré-tratamento hidrotérmico $(\mathrm{PTH})$ foi realizado em reator de aço inoxidável, com capacidade de 5,5 L e controle de agitação e temperatura (PARR Instrument Company, modelo 4848). A palha de cana-de-açúcar teve sua granulometria ajustada empregando um moinho de facas (Willye, modelo SP-30). O conteúdo de água presente nas amostras foi determinado em um determinador de umidade (aquecimento por infravermelho), a $100{ }^{\circ} \mathrm{C}$ em modo automático (Marte Balanças e Aparelhos de Precisão Ltda, modelo ID 50).

Cem gramas (em base seca) de palha de cana-de-açúcar (granulometria de 10 mesh) foram misturadas com água no reator, nas proporções palha/água: 1:5, 1:6,7, 1:10, 1:20 (m/v). Para todas as razões avaliadas, foram aplicadas as mesmas condições de pré-tratamento (temperatura, tempo de reação e agitação): $195^{\circ} \mathrm{C}, 10$ minutos e $200 \mathrm{rpm}$. Ao final da etapa de prétratamento, a fração sólida foi separada da fração líquida por meio de filtração. A fração sólida foi submetida a lavagem com água para a remoção de compostos solubilizados durante a etapa de pré-tratamento até $\mathrm{pH}$ neutro.

$\mathrm{O}$ rendimento mássico $(\mathrm{R})$ obtido após a etapa de pré-tratamento foi determinado por meio da Equação 1.

$$
R=\frac{m_{\text {final }}}{m_{\text {inicial }}} \times 100
$$

onde: $m_{\text {inicial }}=$ massa inicial de material lignocelulósico em base seca $(\mathrm{g}) ; m_{\text {final }}=$ massa final de material lignocelulósico após PTH em base seca $(\mathrm{g})$.

O cálculo do fator de severidade (FS) em cada etapa de pré-tratamento foi realizado seguindo a definição proposta por Overend \& Chornet (1987) [20], conforme a Equação 2.

$$
F S=\log \sum_{i=1}^{n}\left[t * \exp \left(\frac{T_{i}-T_{r e f}}{14,75}\right)\right]
$$


onde: $n$ é o número de intervalos entre as medidas de temperatura durante a etapa prétratamento; $t$ é o tempo médio entre uma medida e outra; $\mathrm{T}_{i}$ é a temperatura do pré-tratamento medida a cada instante de tempo; $T_{r e f}$ é a temperatura de referência, $100^{\circ} \mathrm{C}$.

O FS descreve o efeito combinado de fatores-chave na etapa de pré-tratamento, o tempo e a temperatura. Visto que não é simples avaliar o efeito de cada um desses fatores individualmente, esse fator torna-se bastante importante [20].

\subsubsection{PRÉ-TRATAMENTO ALCALINO}

A etapa de pré-tratamento alcalino (PTA), que consiste na deslignificação da biomassa, ocorreu em sequência ao pré-tratamento hidrotérmico, utilizando-se peróxido de hidrogênio alcalino $\left(\mathrm{H}_{2} \mathrm{O}_{2}, 1 \% \mathrm{v} / \mathrm{v}\right.$, em pH 11,5) como reagente. Essa etapa foi realizada em shaker, a 100 $\mathrm{rpm}$, nas temperaturas de 25,45 e $65^{\circ} \mathrm{C}$, durante 1 hora, com carga de sólidos de $10 \%(\mathrm{~m} / \mathrm{v})$.

\subsubsection{CARACTERIZAÇÃO QUÍMICA DA BIOMASSA}

As amostras de palha de cana-de-açúcar in natura e após cada etapa de pré-tratamento foram caracterizadas utilizando-se a metodologia descrita por Sluiter et al. (2008) [21], modificada por Rocha et al. (1997) [22] e validada por Gouveia et al. (2009) [23]. O material foi moído (20 mesh). A quantidade de extrativos foi determinada para as amostras de palha in natura, visto que após o pré-tratamento os extrativos são solubilizados. O procedimento utiliza um sistema de extração em Soxhlet empregando etanol 95\% como solvente [21].

A quantificação dos carboidratos, ácidos orgânicos e derivados do furano se deu por meio de cromatografia líquida de alta eficiência. A lignina solúvel foi determinada por espectrofotometria a $280 \mathrm{~nm}$. A lignina insolúvel e o teor de cinzas foram obtidos por gravimetria [23].

\subsubsection{PLANEJAMENTO EXPERIMENTAL}

Neste estudo realizou-se um delineamento experimental $2^{2}$, com três repetições no ponto central. O objetivo foi avaliar a influência da razão $m_{\text {palha seca }} / v_{\text {água no }}$ PTH seguido de deslignificação por peróxido de hidrogênio alcalino $\left(\mathrm{H}_{2} \mathrm{O}_{2}\right)$. As variáveis-resposta foram: o teor de celulose corrigido pelo rendimento mássico do PTH (\%) e a conversão de celulose em glicose (\%) determinada após etapa de hidrólise enzimática. As análises estatísticas foram realizadas através do software STATISTICA ${ }^{\circledR}$ (versão 7.0).

A Tabela 1 apresenta o delineamento experimental realizado, com todas as combinações entre os níveis e variáveis estudados. Destaca-se que a escolha dos níveis de cada variável se baseou na literatura $[24,25,26,27]$.

Tabela 1: Matriz do delineamento experimental $2^{2}$ com três repetições no ponto central.

\begin{tabular}{ccc}
\hline Ensaio & $\begin{array}{c}\text { Variável 1: } \\
\text { Razão } \mathbf{m}_{\text {palha seca/vágua no }} \\
\text { PTH (\%) }\end{array}$ & $\begin{array}{c}\text { Variável 2: } \\
\text { Temperatura do } \\
\text { PTA }\left({ }^{\circ} \mathbf{C}\right)\end{array}$ \\
\hline 1 & $1: 20(-)$ & $25(-)$ \\
2 & $1: 6,7(+)$ & $25(-)$ \\
3 & $1: 20(-)$ & $65(+)$ \\
4 & $1: 6,7(+)$ & $65(+)$ \\
5 & $1: 10(0)$ & $45(0)$ \\
6 & $1: 10(0)$ & $45(0)$ \\
7 & $1: 10(0)$ & $45(0)$ \\
\hline
\end{tabular}




\subsubsection{HIDRÓLISE ENZIMÁTICA}

A etapa de hidrólise enzimática foi realizada para as amostras submetidas ao PTH e para as amostras submetidas ao PTH seguido da deslignificação com peróxido de hidrogênio (PTH+PTA). Os ensaios foram realizados em frascos de Erlenmeyer, por 72 horas, à temperatura de $50{ }^{\circ} \mathrm{C}$ e sob agitação de $250 \mathrm{rpm}$ em shaker.

A carga de sólidos utilizada nos experimentos de hidrólise foi de $15 \%(\mathrm{~m} / \mathrm{v})$. O volume de meio reacional foi de $50 \mathrm{~mL}$, composto por material lignocelulósico pré-tratado (em base seca), extrato enzimático (20 FPU/g celulose) e tampão citrato de sódio $50 \mathrm{mM}$ (q.s.p), pH 4,8.

As amostras tiveram seu teor de glicose quantificado por cromatografia líquida de alta eficiência (SCL-10A chromatograph, Shimadzu), com detector de índice de refração (RID10-A, Shimadzu). A coluna utilizada nas análises foi Aminex HPX-87H (Bio-Rad), mantida a $65{ }^{\circ} \mathrm{C}$. Empregou-se como fase móvel solução de $\mathrm{H}_{2} \mathrm{SO}_{4}(5,0 \mathrm{mM})$ na vazão de $0,6 \mathrm{~mL} / \mathrm{min}$. A conversão de celulose em glicose foi calculada através da Equação 3.

$$
\text { Conversão }=\frac{\text { glicose liberada }(g / L)}{\text { concentração de biomassa seca }(g / L) \times f \times 1,11} \times 100
$$

onde: $f$ consiste da fração de celulose presente na biomassa seca; 1,11 corresponde ao fator estequiométrico de conversão de celulose em glicose.

\section{RESULTADOS E DISCUSSÃO}

\subsection{PRÉ-TRATAMENTO HIDROTÉRMICO}

A Tabela 2 apresenta a caracterização química da biomassa in natura e após o PTH realizado.

Tabela 2: Caracterização química da palha in natura e submetida ao PTH $\left(195^{\circ} \mathrm{C}, 10\right.$ minutos, $\left.200 \mathrm{rpm}\right)$ nas diferentes razões palhalágua.

\begin{tabular}{cccccc}
\hline \multirow{2}{*}{ Componente } & Palha In & \multicolumn{4}{c}{ mpalha seca/vágua no PTH $(\mathbf{m} / \mathbf{v})$} \\
\cline { 3 - 6 } & Natura & $\mathbf{1 : 2 0}$ & $\mathbf{1 : 1 0}$ & $\mathbf{1 : 6 , 7}$ & $\mathbf{1 : 5}$ \\
\hline Celulose (\%) & $34,8 \pm 0,2$ & $67,2 \pm 0,8$ & $57,02 \pm 0,01$ & $56,2 \pm 0,8$ & $43,7 \pm 0,1$ \\
Hemicelulose (\%) & $23,0 \pm 0,2$ & $5,56 \pm 0,07$ & $5,80 \pm 0,02$ & $6,18 \pm 0,05$ & $14,2 \pm 0,3$ \\
Lignina total (\%) & $24,1 \pm 0,5$ & $26,7 \pm 0,1$ & $29,4 \pm 0,3$ & $32,3 \pm 1,1$ & $28,4 \pm 0,1$ \\
Cinzas (\%) & $7,1 \pm 0,4$ & $3,5 \pm 0,2$ & $5,9 \pm 0,1$ & $1,80 \pm 0,04$ & $2,3 \pm 0,2$ \\
Extrativos (\%) & $14,9 \pm 0,4$ & - & - & - & - \\
Rendimento mássico (\%) & - & 47,58 & 52,50 & 55,07 & 75,56 \\
Fator de Severidade (-) & - & 4,32 & 4,23 & 4,18 & 4,17 \\
\hline
\end{tabular}

Observa-se na Tabela 2 que o fator de severidade (FS) diminui com o aumento da razão palha/água empregada no pré-tratamento hidrotérmico. Por sua vez o rendimento mássico (R) aumenta à medida que a razão palha/água se eleva.

Sabe-se que à medida que a razão $\mathrm{m}_{\text {palha seca }} / \mathrm{v}_{\text {água }}$ se eleva, haverá menor volume de água para a mesma quantidade de massa seca. Esse fato corrobora para a obtenção de maior rendimento mássico em maiores razões palha/água visto que menor quantidade de íons hidrônios estará disponível para catalisar a hidrólise da biomassa, o que promove pouca solubilização [28]. Em estudos empregando palha de colza submetida a tratamento hidrotérmico catalisado por $\mathrm{H}_{2} \mathrm{SO}_{4}$, $\mathrm{Lu}$ et al. (2009) [29] encontraram resultados semelhantes, nos quais os maiores rendimentos mássicos foram obtidos nas maiores razões palha/água avaliadas. Em razões mais baixas (1:10 e 1:20 m/v), Pérez et al. (2007) [30] não encontraram diferenças significativas nos valores de 
rendimento mássico, o que também foi observado nesse trabalho para razões 1:20, 1:10 e 1:6,7 $(\mathrm{m} / \mathrm{v})$.

Em relação ao fator de severidade, que diminui à medida que a razão $\mathrm{m}_{\text {palha seca }} / \mathrm{Váagua}$ se eleva, isso ocorre devido à presença de menor volume no meio reacional, que aquecerá mais rapidamente. Visto que o fator de severidade é fortemente dependente do tempo e da temperatura, seu valor será menor em reações de maior razão $\mathrm{m}_{\text {palha seca }} / \mathrm{v}_{\text {água }} \mathrm{o}$ que foi observado nesse estudo.

A Figura 1 ilustra os perfis de temperatura obtidos durante a etapa de PTH para cada razão $\mathrm{m}_{\text {palha seca }} / \mathrm{v}_{\text {água }}$. A partir desses dados é que se determina o fator de severidade. De forma geral, os pré-tratamentos apresentaram perfis de severidade semelhantes. Observa-se que em razões menores $(1: 20$ e $1: 10, \mathrm{~m} / \mathrm{v})$, o ensaio levou mais tempo para alcançar a temperatura experimental, visto que há maior volume de água para ser aquecido no reator.

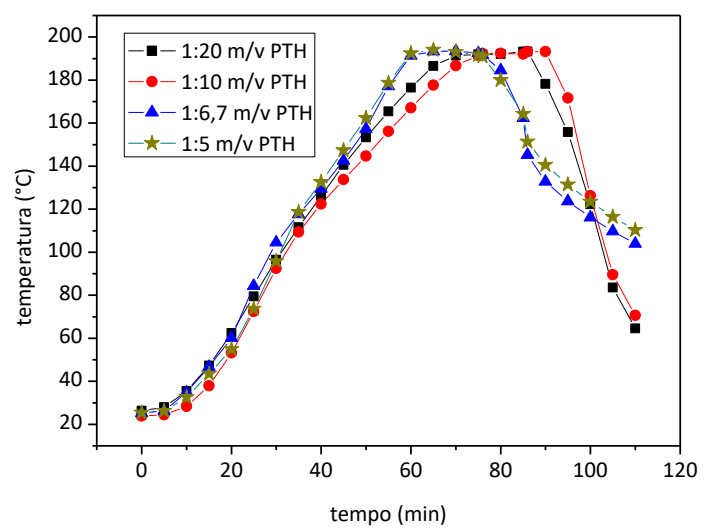

Figura 1: Perfil da temperatura nos diferentes PTH realizados.

A Tabela 3 apresenta a caracterização química das amostras com os valores corrigidos pelo rendimento mássico obtido em cada um dos PTHs.

Tabela 3: Caracterização química com valores corrigidos pelo rendimento do $\mathrm{PTH}\left(195{ }^{\circ} \mathrm{C}, 10 \mathrm{~min}\right.$., 200 rpm).

\begin{tabular}{cccccc}
\hline \multirow{2}{*}{ Componente* } & Palha In & \multicolumn{4}{c}{ Razão mpalha seca/vágua no PTH $(\mathbf{m} / \mathbf{v})$} \\
\cline { 3 - 6 } & Natura & $\mathbf{1 : 2 0}$ & $\mathbf{1 : 1 0}$ & $\mathbf{1 : 6 , 7}$ & $\mathbf{1 : 5}$ \\
\hline Celulose (\%) & $34,8 \pm 0,2$ & 31,97 & 29,94 & 30,94 & 32,98 \\
Hemicelulose (\%) & $23,0 \pm 0,2$ & 2,65 & 3,05 & 3,40 & 10,73 \\
Lignina total (\%) & $24,1 \pm 0,5$ & 12,68 & 15,45 & 17,78 & 21,48 \\
Cinzas (\%) & $7,1 \pm 0,4$ & 1,67 & 3,11 & 0,99 & 1,72 \\
Rendimento másico (\%) & - & 47,58 & 52,50 & 55,07 & 75,56 \\
Fator de Severidade (-) & - & 4,32 & 4,23 & 4,18 & 4,17 \\
\hline
\end{tabular}

*valores corrigidos levando-se em conta o rendimento mássico do PTH.

Analisando os dados da Tabela 3, verifica-se que, para as quatro condições de pré-tratamento avaliadas, o teor de celulose sofreu pouca alteração, observando-se apenas uma pequena perda em relação ao teor de celulose da biomassa in natura $(8,08 \%$ para a razão de $1: 20 ; 13,92 \%$ para a razão de 1:10; 11,04\% para a razão de 1:6,7; e 5,18\% para a razão de 1:5).

Em relação ao teor de hemicelulose e lignina, nota-se que há uma dependência entre o fator de severidade (FS) e a remoção desses dois componentes; à medida que se aumenta o FS, a remoção desses componentes é maior. Nota-se também que a razão de 1:5 (m/v) promoveu remoção não satisfatória em termos de lignina. Nessa mesma razão, a remoção de hemicelulose foi inferior as demais razões estudadas, apesar de ainda representar cerca de 55\%. Esse fato sugere que essa razão não foi eficiente, visto que um dos objetivos principais do PTH é promover remoção da fração hemicelulósica acima de 80\% [5]. Resultados semelhantes foram obtidos por Lu et al. (2009) [29], em que maior fração hemicelulósica foi observada em 
elevadas cargas de sólidos, dentre as avaliadas no pré-tratamento hidrotérmico catalisado por $\mathrm{H}_{2} \mathrm{SO}_{4}$.

Em face da remoção não satisfatória de hemicelulose e lignina, a condição em que se empregou razão de 1:5 m/v no PTH foi descartada para seguir as demais etapas do trabalho.

\subsection{PRÉ-TRATAMENTO ALCALINO SEQUENCIAL AO HIDROTÉRMICO}

A Tabela 4 apresenta os resultados da composição química da palha de cana-de-açúcar após os dois pré-tratamentos sequenciais: hidrotérmico (PTH) e alcalino (PTA) nas condições descritas pelo delineamento experimental.

Tabela 4: Caracterização química das amostras após PTH (195 $\left.{ }^{\circ} \mathrm{C}, 10 \mathrm{~min} ., 200 \mathrm{rpm}\right)$ seguido de PTA.

\begin{tabular}{|c|c|c|c|c|c|}
\hline $\begin{array}{c}\text { Componente } \\
(\%)\end{array}$ & $\begin{array}{l}1: 20 \text { PTH e } \\
25^{\circ} \text { C PTA }\end{array}$ & $\begin{array}{l}\text { 1:20 PTH e } \\
65^{\circ} \text { C PTA }\end{array}$ & $\begin{array}{l}1: 6,7 \text { PTH e } \\
25^{\circ} \text { C PTA }\end{array}$ & $\begin{array}{c}1: 6,7 \text { PTH e } \\
65^{\circ} \text { C PTA }\end{array}$ & $\begin{array}{l}\text { 1:10 PTH e } \\
45^{\circ} \text { C PTA }\end{array}$ \\
\hline Celulose & $51,7 \pm 0,7$ & $61,2 \pm 0,5$ & $64,1 \pm 0,4$ & $67,0 \pm 2,9$ & $62,1 \pm 0,3$ \\
\hline Hemicelulose & $5,6 \pm 0,1$ & $6,7 \pm 0,1$ & $6,2 \pm 0,2$ & $6,4 \pm 0,3$ & $4,51 \pm 0,07$ \\
\hline Lignina total & $22,6 \pm 0,4$ & $25,0 \pm 0,8$ & $22,2 \pm 0,1$ & $20,4 \pm 0,4$ & $21,0 \pm 0,1$ \\
\hline Cinzas & $1,86 \pm 0,02$ & $2,02 \pm 0,04$ & $2,8 \pm 0,1$ & $2,82 \pm 0,04$ & $5,1 \pm 0,2$ \\
\hline $\begin{array}{c}\text { Rendimento } \\
\text { PTA }\end{array}$ & 80,01 & 75,51 & 81,57 & 73,93 & 72,09 \\
\hline
\end{tabular}

Para análise das condições estudadas nos pré-tratamentos PTH+PTA, procedeu-se de forma análoga aos ensaios de PTH. São apresentados, na Tabela 5, os resultados de composição química levando-se em conta o rendimento mássico das duas etapas realizadas (PTH+PTA).

Tabela 5: Composição química levando-se em conta o rendimento mássico dos pré-tratamentos PTH e PTA.

\begin{tabular}{|c|c|c|c|c|c|}
\hline $\begin{array}{c}\text { Componente* } \\
(\%)\end{array}$ & $\begin{array}{l}\text { 1:20 PTH e } \\
25^{\circ} \text { C PTA }\end{array}$ & $\begin{array}{l}\text { 1:20 PTH e } \\
65^{\circ} \mathrm{C} \text { PTA }\end{array}$ & $\begin{array}{l}\text { 1:6,7 PTH e } \\
25^{\circ} \text { C PTA }\end{array}$ & $\begin{array}{l}1: 6,7 \text { PTH e } \\
65^{\circ} \text { C PTA }\end{array}$ & $\begin{array}{l}\text { 1:10 PTH e } \\
45^{\circ} \text { C PTA }\end{array}$ \\
\hline Celulose & 19,68 & 21,99 & 28,79 & 27,28 & 23,50 \\
\hline Hemicelulose & 2,13 & 2,42 & 2,79 & 2,60 & 1,71 \\
\hline Lignina total & 8,60 & 9,20 & 9,97 & 8,31 & 7,95 \\
\hline Cinzas & 0,71 & 0,68 & 1,26 & 1,15 & 1,93 \\
\hline $\begin{array}{l}\text { Rendimento } \\
\text { mássico } \\
\text { PTH+PTA }\end{array}$ & 38,07 & 35,93 & 44,92 & 40,71 & 37,85 \\
\hline \multicolumn{6}{|c|}{ *valores corrigidos levando-se em conta o rendimento mássico do PTH+PTA. } \\
\hline \multicolumn{6}{|c|}{$\begin{array}{l}\text { Observa-se na Tabela } 5 \text { que maiores teores de celulose foram obtidos com a razão 1:6,7 no } \\
\text { PTH, em combinação com as temperaturas dos níveis inferior e superior do PTA. O teor de } \\
\text { hemicelulose, lignina e cinzas não sofreu variações expressivas quando comparados entre si. } \\
\text { As Figuras } 2,3 \text { e } 4 \text { apresentam o percentual de remoção dos componentes principais } \\
\text { (celulose, hemicelulose e lignina) da biomassa para as três razões palha/água avaliadas (1:20; } \\
\text { 1:10; } 1: 6,7) \text {, respectivamente. }\end{array}$} \\
\hline
\end{tabular}




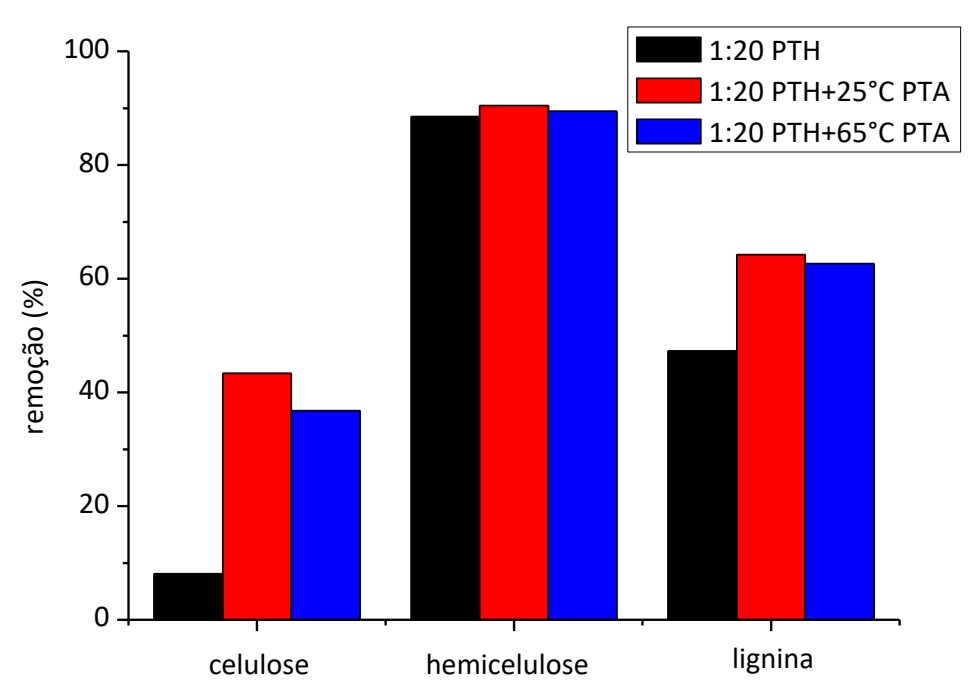

Figura 2: Remoção dos principais componentes da biomassa na razão 1:20 no PTH seguido ou não de deslignificação.

Observa-se pela Figura 2 que o PTH+PTA promoveu remoção considerável de lignina: $64,24 \%$ na condição de $1: 20$ no PTH+ $25^{\circ} \mathrm{C}$ no PTA e $62,64 \%$ na condição de $1: 20$ no PTH+ $65{ }^{\circ} \mathrm{C}$ no PTA, frente a $47,26 \%$ na condição onde ocorreu apenas o PTH. A remoção de hemicelulose após PTA praticamente não se alterou comparando-se com a condição onde ocorreu apenas o PTH. Isso se deve ao fato de que além de o PTA não apresentar a característica de remoção da fração hemicelulósica, é notável que bastante hemicelulose foi removida no $\mathrm{PTH}$, diminuindo de forma expressiva o percentual remanescente de hemicelulose passível a remoção pelo PTA. Já em termos de celulose, houve perda considerável dessa fração, o que não é desejado. 43,37\% de perda de material celulósico foi detectada na condição de 1:20 no PTH $+25{ }^{\circ} \mathrm{C}$ no PTA e $36,78 \%$ na condição de $1: 20$ no PTH+ $65{ }^{\circ} \mathrm{C}$ no PTA. No ensaio onde ocorreu somente PTH houve remoção de apenas $8,08 \%$ de celulose. Para essa razão $\mathrm{m}_{\text {palha }}$ seca/vágua conclui-se, portanto, que a deslignificação não se mostrou eficiente visto que removeu parte da lignina, porém promoveu severa perda de celulose.

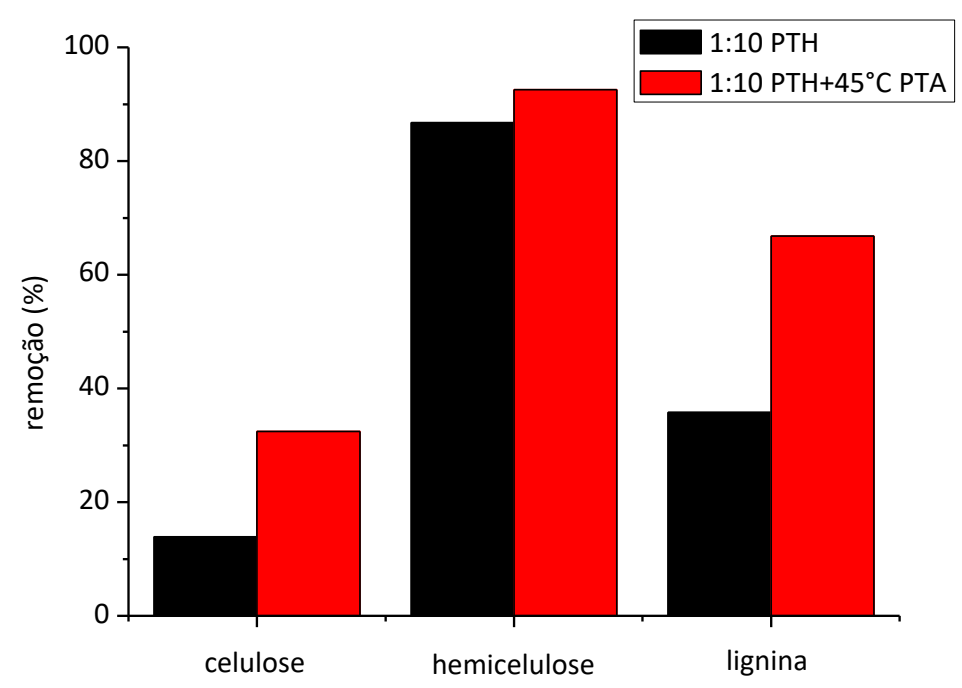

Figura 3: Remoção dos principais componentes na razão 1:10 no PTH seguido ou não de deslignificação. 
A Figura 3 apresenta os percentuais de remoção dos 3 componentes principais da biomassa lignocelulósica na condição de 1:10 no PTH seguido ou não de deslignificação. Analisando seus resultados, nota-se que $66,81 \%$ da lignina foi removida no ensaio onde ocorreu PTH+PTA, frente a $35,78 \%$ no ensaio onde ocorreu apenas o pré-tratamento hidrotérmico (PTH). Maior percentual de remoção de hemicelulose também foi encontrado para o ensaio seguido de deslignificação: $92,57 \%$, frente a $86,74 \%$ no ensaio onde foi realizado apenas PTH. A perda de material celulósico foi de $32,42 \%$ na condição de PTH+PTA e de $13,93 \%$ na condição onde ocorreu apenas PTH.

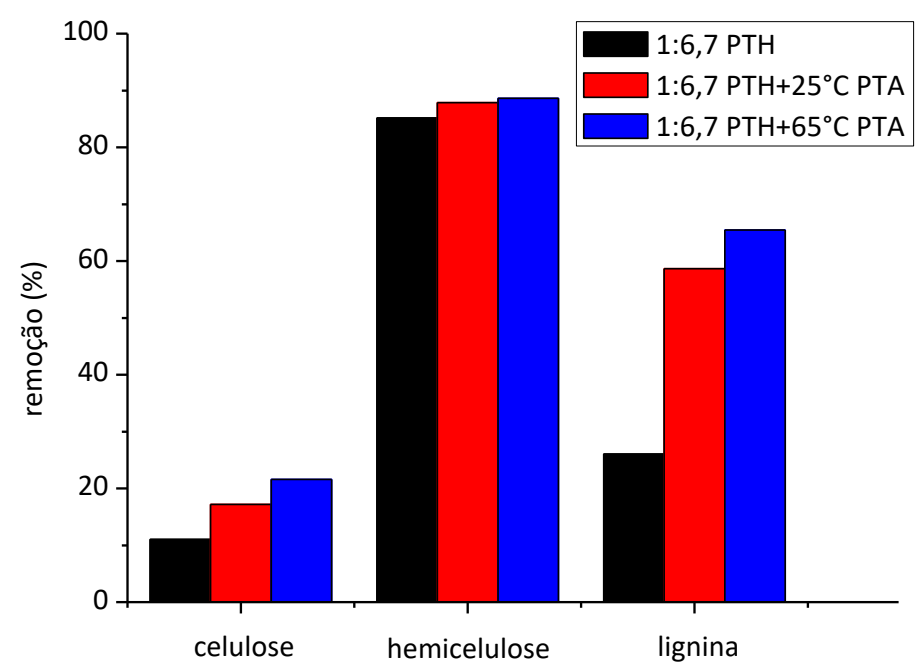

Figura 4: Remoção dos principais componentes na razão 1:6,7 no PTH seguido ou não de deslignificação.

Ao avaliar os ensaios que ocorreram com a razão de 1:6,7 no PTH, observa-se (Figura 4) que o ensaio à 1:6,7 no PTH e $25{ }^{\circ} \mathrm{C}$ no PTA promoveu remoção de 58,63\% de lignina; já o ensaio à 1:6,7 no PTH e $65{ }^{\circ} \mathrm{C}$, promoveu $65,43 \%$ de remoção de lignina, frente a $26,06 \%$ no ensaio em que ocorreu apenas o pré-tratamento hidrotérmico. Da mesma forma que na condição de 1:20, a remoção de hemicelulose adicional não foi expressiva quando comparada com a condição onde ocorreu apenas o PTH. Em termos de celulose, a perda detectada para as condições que sofreram pré-tratamento alcalino foi mais branda comparando-se com a condição de 1:20: $17,18 \%$ para o ensaio à $1: 6,7$ no $\mathrm{PTH}$ e $25^{\circ} \mathrm{C}$ no PTA e $21,56 \%$ para o ensaio à $1: 6,7$ no PTH e $65{ }^{\circ} \mathrm{C}$. Na condição onde ocorreu apenas o PTH, houve perda de $11,03 \%$ de celulose.

\subsection{HIDRÓLISE ENZIMÁTICA}

Após os pré-tratamentos, todas as frações sólidas geradas foram submetidas à hidrólise enzimática para verificar, em termos de conversão de celulose em glicose, as condições de maior potencial de produção de etanol de segunda geração. Os resultados estão apresentados nas Figuras 5, 6 e 7. 


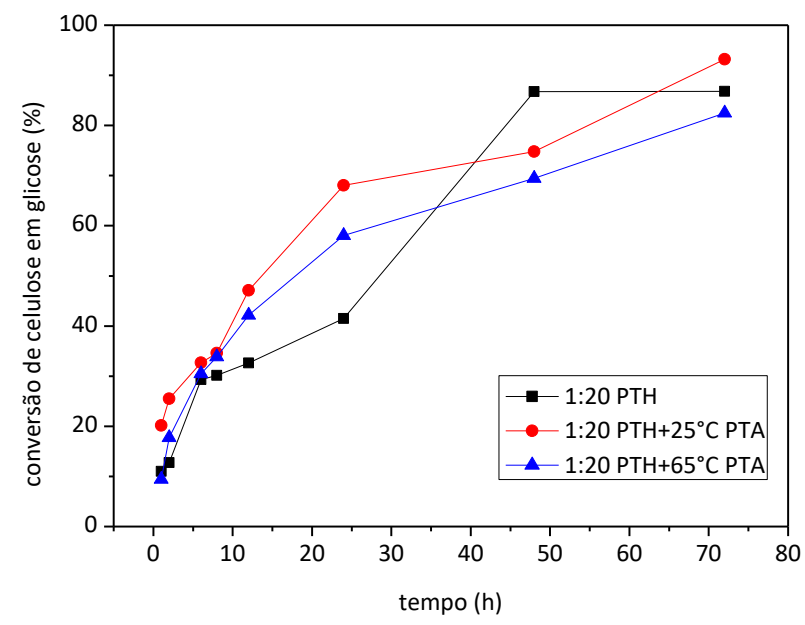

Figura 5: Conversão de celulose em glicose durante 72 horas de hidrólise enzimática realizada com biomassa pré-tratada na razão palhalágua 1:20 para o PTH e 1:20 para os PTH + PTA. Experimentos de hidrólise realizados com carga de sólidos de $15 \%(\mathrm{~m} / \mathrm{v})$.

Nota-se pela Figura 5 que as conversões obtidas com razão palha/água de 1:20 no PTH, seguido ou não de tratamento alcalino, apresentaram perfis semelhantes; a conversão de celulose em glicose se elevou ao longo do tempo reacional de 72 horas. A biomassa que foi submetida ao pré-tratamento PTH + PTA a $65^{\circ} \mathrm{C}$ foi a que demonstrou menor conversão ao longo das 72 horas de hidrólise enzimática, $82,49 \%$. Este resutado foi bem próximo daquele em que se empregou biomassa submetida apenas ao PTH, 86,79\%. A biomassa que apresentou maior conversão foi a que passou por PTH seguido de PTA a $25^{\circ} \mathrm{C}$, alcançando $93,19 \%$.

Esperava-se que as biomassas submetidas à deslignificação apresentassem maiores conversões na etapa de hidrólise, visto que a presença da lignina dificulta o acesso das enzimas à celulose. Porém, esse resultado não foi constatado para todos os ensaios realizados neste trabalho.

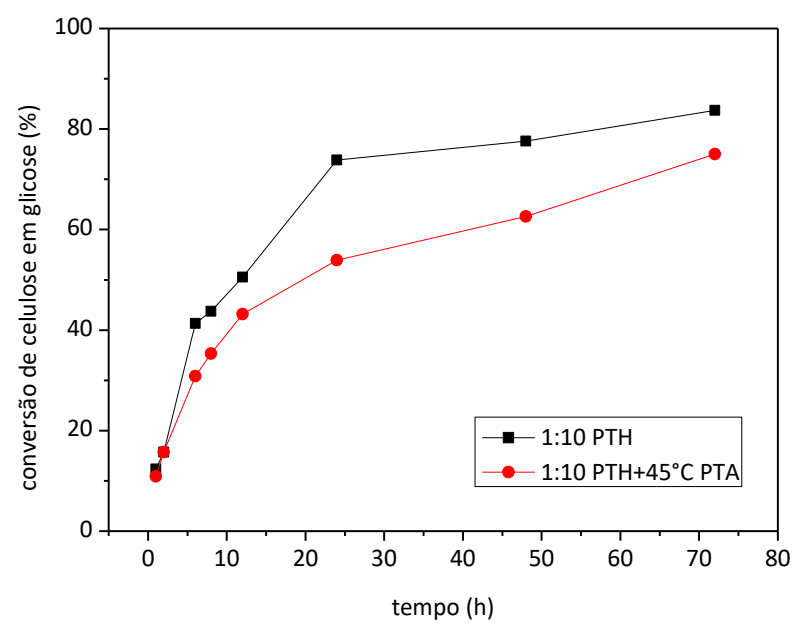

Figura 6: Conversão de celulose em glicose durante 72 horas de hidrólise enzimática com biomassa prétratada na razão palha/água 1:10 no pré-tratamento hidrotérmico $(P T H)$ e 1:10 no PTH+PTA. Experimentos de hidrólise realizados com carga de sólidos de $15 \%(\mathrm{~m} / \mathrm{v})$. 
A Figura 6 mostra que maior conversão na hidrólise foi alcançada no ensaio em que a biomassa foi submetida apenas ao PTH: $83,72 \%$, frente a 75,01\% (média da triplicata) da biomassa submetida ao pré-tratamento sequencial PTH+PTA.

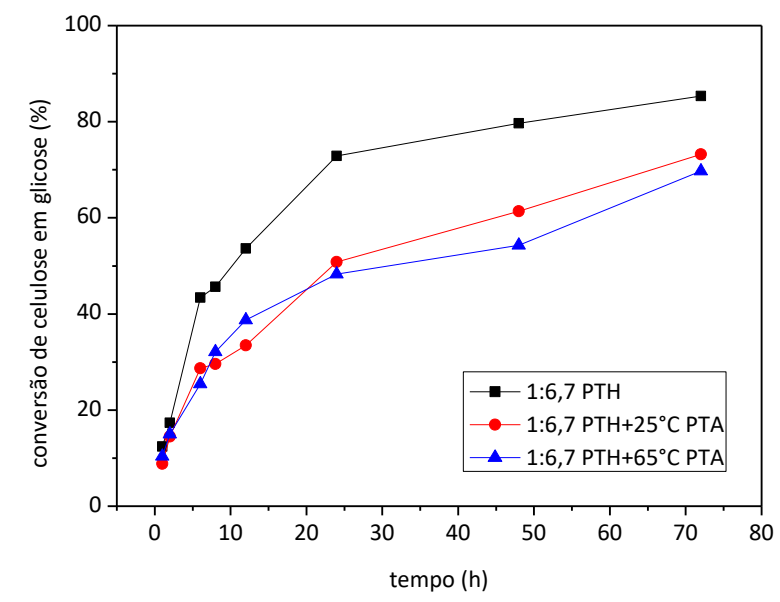

Figura 7: Conversão de celulose em glicose durante 72 horas de hidrólise enzimática com biomassa prétratada na razão palha-agua 1:6,7 no pré-tratamento hidrotérmico $(P T H)$ e 1:6,7 no PTH+PTA.

Experimentos de hidrólise realizados com carga de sólidos de $15 \%(\mathrm{~m} / \mathrm{v})$.

A Figura 7 indica perfil semelhante à condição de 1:20 no PTH. Para o ensaio de PTH+PTA a $65{ }^{\circ} \mathrm{C}$, foi detectada a menor conversão entre os ensaios realizados $(69,73 \%)$. O ensaio em que se empregou biomassa submetida aos pré-tratamentos PTH+PTA a $25{ }^{\circ} \mathrm{C}$ apresentou $73,23 \%$ de conversão. Já o ensaio onde a biomassa sofreu apenas o PTH, a conversão foi de $85,36 \%$, a maior entre as avaliadas na razão 1:6,7 no PTH.

Nota-se que, de forma geral, as biomassas submetidas apenas a PTH apresentaram maior conversão na etapa de hidrólise. Esse fato pode ser atribuído à eficiência da enzima utilizada, convertendo de forma eficiente a celulose em glicose após o PTH, ainda que na presença de maior teor de lignina (para as biomassas que não sofreram PTA). Portanto, em analogia aos ensaios que não sofreram deslignificação, não houve divergência significativa entre os seus resultados e os resultados obtidos após etapa de deslignificação, visto que nos ensaios em que a biomassa não sofreu tratamento alcalino foram obtidos rendimentos similares. Viikari et al., (2012) [19] afirmaram que a hidrólise mostra-se significativamente melhorada após PTH, a despeito da presença da lignina, efeito que esteve em acordo com os resultados obtidos nesse trabalho.

A literatura relata ainda que a fração de lignina, ao ser submetida a elevadas temperaturas, pode sofrer um processo denominado repolimerização, sucedido quando a lignina se precipita gerando assim a chamada pseudo-lignina, que apresenta um efeito ainda mais negativo sobre os carboidratos que a própria lignina intrínseca à biomassa. Esse efeito caracteriza-se por aumentar a reatividade da lignina remanescente e assim dificultar o aproveitamento dos açúcares pela enzima aplicada posteriormente na etapa de sacarificação [31, 32, 33]. Em face dos resultados obtidos na hidrólise enzimática, sugere-se que essa consequência negativa foi detectada nos ensaios que sofreram deslignificação.

\subsection{ANÁLISE ESTATÍSTICA}

A Tabela 6 apresenta a matriz do planejamento experimental realizado, com todas as combinações entre os fatores e os resultados obtidos para as variáveis-resposta. 
Tabela 6: Matriz de planejamento fatorial e as variáveis-resposta.

\begin{tabular}{ccccc}
\hline Ensaios & $\begin{array}{c}\text { Variável 1: } \\
\text { Razão mpalha } \\
\text { seca/vágua no PTH } \\
(\boldsymbol{\%})\end{array}$ & $\begin{array}{c}\text { Variável 2: } \\
\text { Temperatura do } \\
\text { PTA }\left({ }^{\circ} \mathbf{C}\right)\end{array}$ & $\begin{array}{c}\text { Variável } \\
\text { Resposta: } \\
\text { Teor de celulose } \\
\text { corrigido* }(\%)\end{array}$ & $\begin{array}{c}\text { Variável } \\
\text { Resposta: } \\
\text { Conversão de } \\
\text { celulose em } \\
\text { glicose }(\%)\end{array}$ \\
\hline 1 & $1: 20(-)$ & $25(-)$ & 19,70 & 93,19 \\
2 & $1: 6,7(+)$ & $25(-)$ & 28,80 & 73,23 \\
3 & $1: 20(-)$ & $65(+)$ & 21,99 & 82,49 \\
4 & $1: 6,7(+)$ & $65(+)$ & 27,28 & 69,73 \\
5 & $1: 10(0)$ & $45(0)$ & $23,10 \pm 0,06$ & $74,90 \pm 0,14$ \\
6 & $1: 10(0)$ & $45(0)$ & $23,20 \pm 0,06$ & $75,29 \pm 0,14$ \\
7 & $1: 10(0)$ & $45(0)$ & $22,98 \pm 0,06$ & $74,84 \pm 0,14$ \\
\hline
\end{tabular}

*corrigido pelo rendimento mássico do PTH.

Observa-se na Tabela 6 que maiores teores de celulose foram encontrados com a maior razão

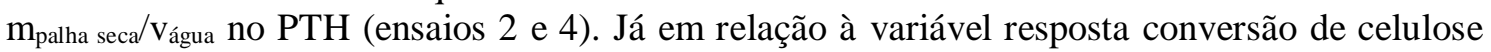
em glicose, o ensaio 1, no qual a razão no PTH foi de 1:20, apresentou maior conversão. As Figuras 8 e 9 apresentam, respectivamente, os gráficos de pareto e as superfícies de resposta gerados para as duas variáveis-resposta avaliadas.
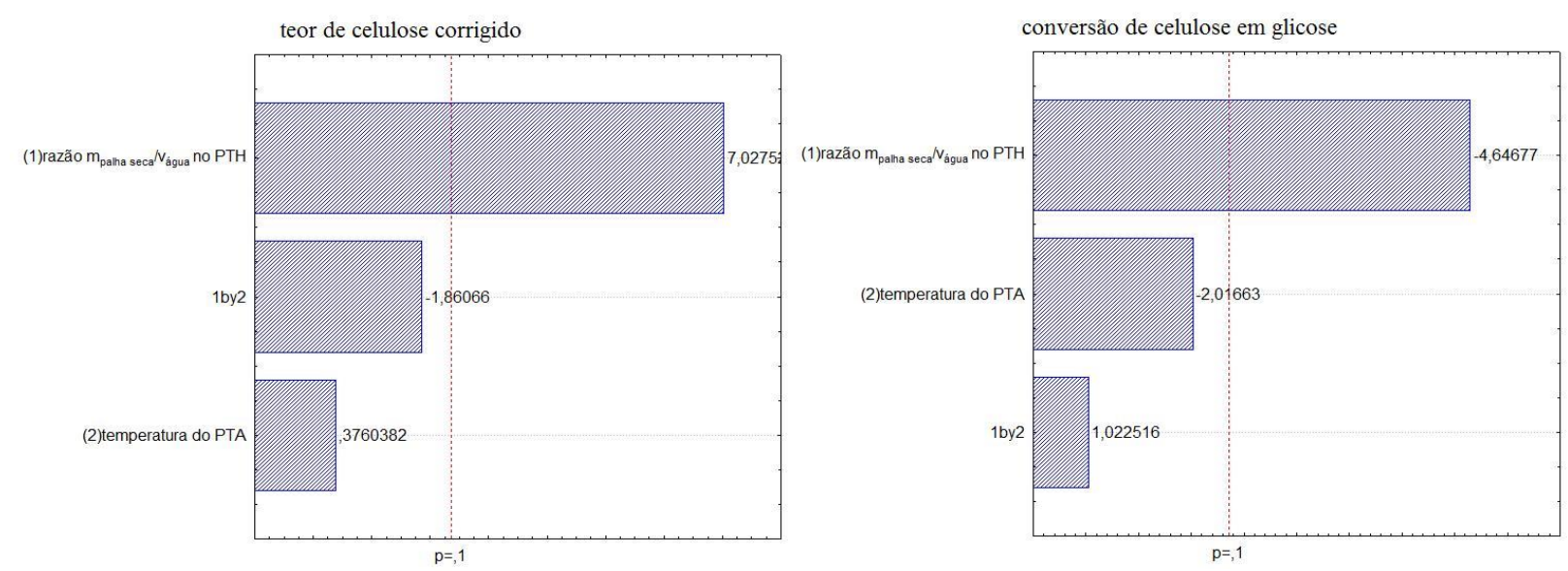

Figura 8: Gráficos de pareto para as duas variáveis-resposta avaliadas (teor de celulose corrigido e conversão de celulose em glicose). 

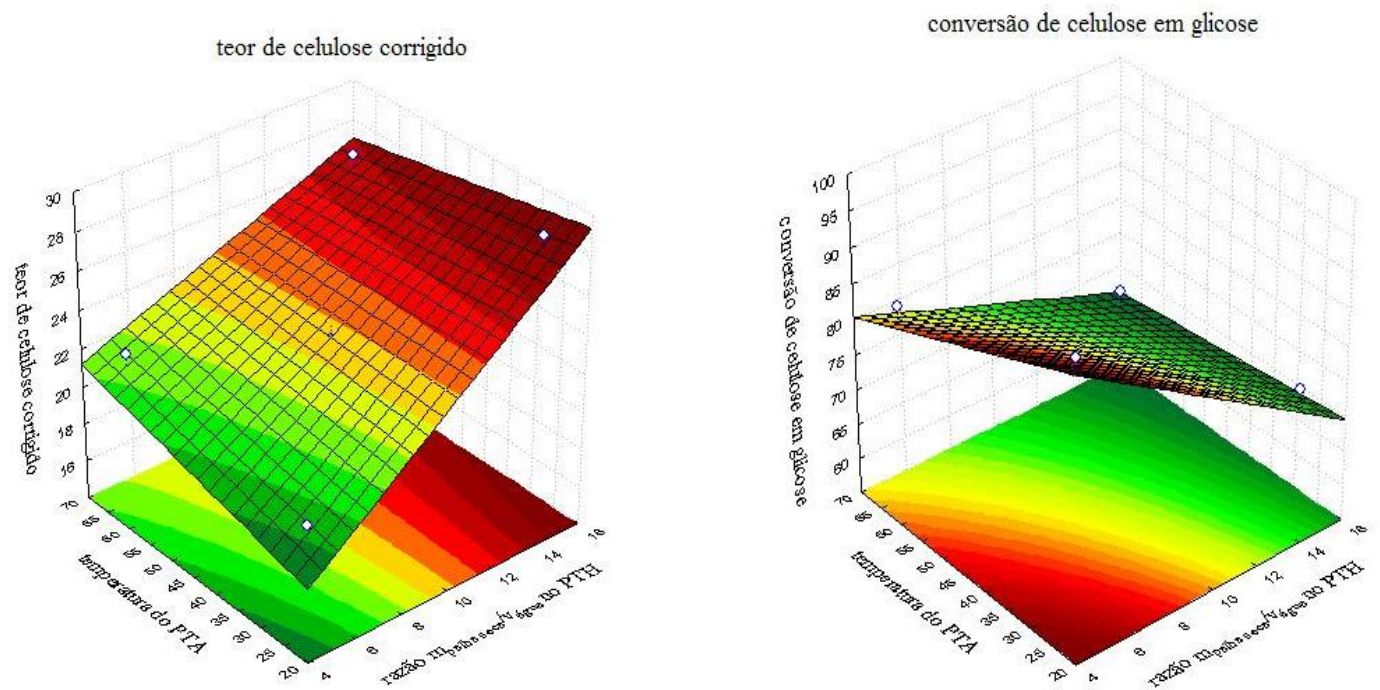

Figura 9: Superfícies de resposta geradas para as duas variáveis-resposta avaliadas: (a) teor de celulose corrigido; (b) conversão de celulose a glicose.

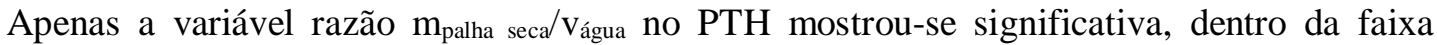
estudada, a um nível de $90 \%$ de confiança. As Equações 4 e 5 apresentam os coeficientes do modelo matemático, levando-se em conta apenas o fator significativo. A variável-resposta "teor de celulose corrigido (c)" é representada pela Equação 4 e a variável-resposta "conversão de celulose em glicose (g)" está representada na Equação 5. O " $x$ " em ambas as equações indica a razão $\mathrm{m}_{\text {palha seca }} / \mathrm{v}_{\text {água }}$ no PTH. Ambas as equações apresentaram coeficiente de determinação ajustado $\left(\mathrm{R}^{2}\right)=1$.

$$
\begin{aligned}
& c=11,95+1,15^{*} x \\
& g=110,11-2,45^{*} x
\end{aligned}
$$

A análise de variância para a variável-resposta 'teor de celulose corrigido' aponta que o fator F (teste de significância que relaciona a média quadrática da regressão/média quadrática do resíduo) calculado foi 9,83 vezes maior que o valor $\mathrm{F}$ tabelado, a um nível de $90 \%$ de confiança. Já para a variável resposta 'conversão de celulose em glicose', o F calculado foi 4,95 vezes superior ao valor $\mathrm{F}$ tabelado, indicando ajuste dos dados ao modelo linear [34, 35].

Observando as Figuras 8 e 9, e as Equações 4 e 5, nota-se que em relação às duas variáveisresposta, o modelo ajustou-se bem aos dados experimentais. Por análise da variável 'teor de celulose corrigido' nas Figuras 8 e 9, o fator 'razão $\mathrm{m}_{\text {palha seca }} /$ água no PTH' apresentou-se estatisticamente significativo a um nível de $90 \%$ de confiança, indicando que maiores teores de celulose são encontrados em elevadas razões palha/água. Em relação à variável 'conversão de celulose em glicose', o mesmo fator da variável-resposta anterior (porém com efeito inverso), razão $\mathrm{m}_{\text {palha seca }} / \mathrm{v}_{\text {água }}$ no $\mathrm{PTH}$, apresentou-se significativo dentro da faixa estudada, indicando que maior conversão de celulose em glicose foi alcançada ao se trabalhar no nível inferior desse fator.

Para ambas as variáveis-resposta estudadas, os demais fatores (temperatura do PTA e a interação entre os dois fatores) não se mostraram significativos dentro da faixa estudada. A possível explicação está associada aos níveis de temperatura empregados no PTA, que podem não ter sido suficientes para provocar efeito significativo nas variáveis-resposta avaliadas. 
O estudo de parâmetros operacionais associados à etapa de pré-tratamento da palha de canade-açúcar apresentado neste trabalho mostrou-se útil para elucidar o comportamento da biomassa lignocelulósica estudada durante o pré-tratamento hidrotérmico seguido de deslignificação. Foi possível constatar que a hidrólise enzimática não foi melhorada após o prétratamento alcalino, logo, diante da elevada eficiência do pré-tratamento hidrotérmico identificada no estudo, custos operacionais concernentes à etapa alcalina podem ser descartados. Dessa forma, a produção de etanol $2 \mathrm{G}$ pode ser otimizada em termos econômicos sem causar prejuízos relacionados a conversão de celulose em glicose.

\section{CONCLUSÃO}

Tendo em vista que o pré-tratamento é uma etapa fundamental no processo de produção do etanol de segunda geração, torna-se importante estudar essa etapa com objetivo de otimizar as condições operacionais empregadas.

O trabalho avaliou o emprego de diferentes razões palha/água no pré-tratamento hidrotérmico (1:20 até 1:6,7), bem como diferentes temperaturas no pré-tratamento alcalino (25 a $65{ }^{\circ} \mathrm{C}$ ), com realização de experimentos de sacarificação enzimática para todas as biomassas submetidas tanto ao pré-tratamento hidrotérmico $(\mathrm{PTH})$ quanto aos pré-tratamentos sequenciais, hidrotérmico e alcalino (PTH+PTA). O estudo mostrou que a etapa de deslignificação alcalina não promoveu efetiva melhora na conversão de celulose em glicose, o que não justifica sua realização. Porém, o pré-tratamento hidrotérmico mostrou-se promissor, alcançando conversões na etapa de hidrólise acima de 85\%, especialmente para as razões palha/água de 1:6,7 e 1:20.

$\mathrm{O}$ planejamento experimental mostrou que o fator razão $\mathrm{m}_{\text {palha seca }} / \mathrm{v}_{\text {água }}$ no PTH se apresentou estatisticamente significativo dentro da faixa estudada, indicando que maior teor de celulose foi obtido ao se trabalhar no nível superior desse fator. Efeito inverso, porém, foi detectado para a variável-resposta conversão de celulose em glicose, visto que maior conversão foi obtida ao se trabalhar no nível inferior do fator razão $\mathrm{m}_{\text {palha seca }} / \mathrm{v}_{\text {água }}$ no $\mathrm{PTH}$.

\section{AGRADECIMENTOS}

A CAPES - Coordenação de Aperfeiçoamento de Pessoal de nível Superior pelo apoio financeiro. Ao projeto "Casadinho" / PROCAD, CNPq/CAPES (n552595/2011-0). Ao Programa de Pós-Graduação em Engenharia Química - PPG-EQ/UFSCar.

\section{REFERÊNCIAS BIBLIOGRÁFICAS}

1. Gonzalez R, Daystar J, Lett M, Treasure T, Jameel H, Venditti R, Phillips R. Economics of cellulosic ethanol production in a thermochemical pathway for softwood, hardwood, corn stover and switchgrass. Fuel Process Technol. 2012 Feb; 94 (1): 113-2, doi: 10.1016/j.fuproc.2011.10.003.

2. Sanchez A, Sevilla-Güitrón V, Magaña G, Gutierrez L. Parametric analysis of total costs and energy efficiency of 2G enzymatic ethanol production. Fuel. 2013 Nov; 113: 165-79, doi:10.1016/j.fuel.2013.05.034.

3. Santos FA, Queiróz JH, Colodette JL, Manfredi M, Queiroz MELR, Caldas CS, Soares FEF. Otimização do pré-tratamento hidrotérmico da palha de cana-de-açúcar visando à produção de etanol celulósico. Quim Nova. 2014; 37 (1): 56-62, doi: 10.1590/S0100-40422014000100011.

4. Pereira SC, Maehara L, Machado CMM, Farinas CS. 2G ethanol from the whole sugarcane lignocellulosic biomass. Biotechnol Biofuels.2015 Mar; 8 (44): 1-16, doi: 10.1186/s13068-015-02240 .

5. Pratto B. Hidrólise enzimática da palha de cana-de-açúcar: estudo cinético e modelagem matemática semi-mecanística. [dissertação]. São Carlos - SP (Brasil): Universidade Federal de São Carlos; 2015. $141 \mathrm{p}$.

6. Corrêa LJ. Estudos de melhorias no processo de hidrólise enzimática de biomassas para produção de etanol. [tese]. São Carlos - SP (Brasil): Universidade Federal de São Carlos; 2016. 132 p. 
7. Oliveira LRM, Nascimento VM, Gonçalves AR, Rocha GJM. Combined process system for the production of bioethanol from sugarcane straw. Ind Crops Prod. 2014 Jul; 58: 1-7, doi: 10.1016/j.indcrop.2014.03.037.

8. Pereira SC, Maehara L, Machado CMM, Farinas CS. Physical-chemical-morphological characterization of the whole sugarcane lignocellulosic biomass used for $2 \mathrm{G}$ ethanol production by spectroscopy and microscopy techniques. Renew Energ. 2016 Mar; 87 (1): 607-17, doi: 10.1016/j.renene.2015.10.054.

9. Pratto B, Souza RBA, Sousa Jr R, Cruz AJG. Enzymatic Hydrolysis of Pretreated Sugarcane Straw: Kinetic Study and Semi-Mechanistic Modeling. Appl Biochem Biotechnol. 2016 Apr; 178 (7): 143044, doi: 10.1007/s12010-015-1957-8.

10. Lennartsson PR, Erlandsson P, Taherzadeh J. Integration of the first and second generation bioethanol processes and the importance of by-products. Bioresour Technol. 2014 Aug; 165: 3-8, doi: 10.1016/j.biortech.2014.01.127.

11. Abdullah R, Ueda K, Saka S. Hydrothermal decomposition of various crystalline celluloses as treated by semi-flow hot-compressed water. J Wood Sci. 2014Aug; 60 (4): 278-86, doi:10.1007/s10086-0141401-7.

12. Boussarsar H, Rogé B, Mathlouthi M. Optimization of sugarcane bagasse conversion by hydrothermal treatment for the recovery of xylose. Bioresour Technol. 2009 Dec; 100 (24): 6537-42, doi: 10.1016/j.biortech.2009.07.019.

13. Da Cruz SH, Nichols NN, Dien BS, Saha BC, Cotta MA. Hydrothermal pretreatment of sugarcane bagasse using response surface methodology improves digestibility and ethanol production by SSF. J Ind Microbiol Biotechnol. 2012 Mar; 39 (3): 439-47, doi:10.1007/s10295-011-1051-3.

14. Silva VFN, Arruda PV, Felipe MGA, Gonçalves AR, Rocha GJM.Fermentation of cellulosic hydrolysates obtained by enzymatic saccharification of sugarcane bagasse pretreated by hydrothermal processing. J Ind Microbiol Biotechnol. 2011 Jul;38 (7): 809-17, doi:10.1007/s10295-010-0815-5.

15. Xiao LP, Shi ZJ, Xu F, Sun RC. Hydrothermal treatment and enzymatic hydrolysis of Tamarix ramosissima: Evaluation of the process as a conversion method in a biorefinery concept. Bioresour Technol. 2013 May; .135: 73-81. doi:10.1016/j.biortech.2012.08.143.

16. Banerjee G, Car S, Liu T, Williams DL, Meza SL, Walton JD, Hodge DB. Scale-up and integration of alkaline hydrogen peroxide pretreatment, enzymatic hydrolysis, and ethanolic fermentation. Biotechnol Bioeng. 2012 Apr; 109 (4): 922-31, doi: 10.1002/bit.24385.

17. Corrêa LJ, Badino AC, Cruz AJG. Mixing design for enzymatic hydrolysis of sugarcane bagasse: methodology for selection of impeller configuration. Bioprocess Biosyst Eng. 2016aFeb; 39 (2):28594, doi: 10.1007/s00449-015-1512-6.

18. Corrêa LJ, Badino AC, Cruz AJG. Power consumption evaluation of different fed-batch strategies for enzymatic hydrolysis of sugarcane bagasse. Bioprocess Biosyst Eng. 2016b May; 39 (5): 825-33, doi: 10.1007/s00449-016-1562-4.

19. Viikari L, Vehmaanperä J, Koivula A. Lignocellulosic ethanol: From science to industry. Biomass Bioenerg. 2012 Nov; 46: 13-24, doi: 10.1016/j.biombioe.2012.05.008.

20. Overend RP, Chornet E. Fractionation of lignocellulosics by steam-aqueous pretreatments. Philos. T. R. Soc. A.1987 Apr; 321 (1561): 523-36, doi: 10.1098/rsta.1987.0029.

21. Sluiter A, Hames B, Ruiz R, Scarlata C, Sluiter J, Templeton D, Crocker D. Determination of structural carbohydrates and lignin in biomass. Technical Report NREL/TP-510-42618. 2008. 18 p.

22. Rocha G, Silva F, Curvelo A, Araújo G. A fast and accurate method for determination of cellulose and polyoses by HPLC. Fifth brazilian Symp. Chem. lignins other wood components. 1997. 6 p.

23. Gouveia ER, Do Nascimento RT, Souto-Maior AM. Validação de metodologia para a caracterização química de bagaço de cana-de-açúcar. Quim Nova.2009 Jul; 32 (6) 1500-3.

24. Gonçalves FA, Ruiz HA, Nogueira CC, Santos ES, Teixeira JA, Macedo GR. Comparison of delignified coconuts waste and cactus for fuel-ethanol production by the simultaneous and semisimultaneous saccharification and fermentation strategies. Fuel 2014 Sep; 131: 66-76, doi: 10.1016/j.fuel.2014.04.021.

25. Díaz AP, Blandino A, Belleli C, Caro I. An effective process for pretreating rice husk to enhance enzyme hydrolysis. Ind Eng Chem Res.2014 Jun; 53 (27) 10870-5, doi: 10.1021/ie501354r.

26. Ayeni AO, Omoleye JA, Mudliar S, Hymore FK, Pandey RA. Utilization of lignocellulosic waste for ethanol production: Enzymatic digestibility and fermentation of pretreated shea tree sawdust. Korean J Chem Eng. 2014 Jul; 31 (7): 1180-6, doi: 10.1007/s11814-014-0026-2.

27. Wang Q,Wang Z,Shen F,Hu J,Sun F,Lin L,Yang G,Zhang Y,Deng S. Pretreating lignocellulosic biomass by the concentrated phosphoric acid plus hydrogen peroxide (PHP) for enzymatic hydrolysis: Evaluating the pretreatment flexibility on feedstocks and particle sizes. Bioresour Technol. 2014 Aug; 166: 420-8, doi: 10.1016/j.biortech.2014.05.088. 
28. Martínez JDA. Modelagem cinética da hidrólise enzimática da palha de cana-de-açúcar pré-tratada hidrotermicamente. [dissertação]. Rio de Janeiro (Brasil): Universidade Federal do Rio de Janeiro; 2014. 107 p.

29. Lu X, Zhang Y, Angelidaki I. Optimization of $\mathrm{H}_{2} \mathrm{SO}_{4}$ - catalyzed hydrothermal pretreatment of repessed straw for bioconversion to ethanol: Focusing on pretreatment at high solids content. Bioresour. Technol. 2009 Jun; 100 (12): 3048-53, doi: 10.1016/j.biortech.2009.01.008.

30. Pérez JA, González A, Oliva JM, Ballesteros I, Manzanares P. Effect of process variables on liquid hot water pretreatment of wheat straw for bioconversion to fuel-ethanol in a batch reactor. J Chem Technol Biot. 2007 Oct; 82 (10): 929-38, doi: 10.1002/jctb. 1765.

31. Leschinsky M, Zuckerstätter G, Weber HK, Patt R, Sixta H. Effect of autohydrolysis of Eucalyptus globulus wood in lignin structure. Part 1: Comparison of different lignins fractions formed during water prehydrolysis. Holzforschung. 2008 Nov; 62(6): 645-2, doi: 10.1515/HF.2008.133.

32. Borrega M, Nieminen K, Sixta H. Effects of hot water extraction in a batch reactor on the delignification of birch wood. Bioresources. 2011 Jun; 6(2): 1890-903, doi: 10.15376/biores.6.2.18901903.

33. Leschinsky M, Sixta H, Patt, R. Detailed mass balances of the autohydrolysis of Eucalyptus globulus at $170^{\circ} \mathrm{C}$. Bioresources. 2009 May; 4(2): 687-703.

34. Neto BB, Scarmínio IS, Bruns RE. Como fazer experimentos. Pesquisa e desenvolvimento na ciência e na indústria. Brasil: $2^{\text {a }}$ Ed., Editora Unicamp - Campinas, SP, 2001. 412 p.

35. Rodrigues MI, Iemma AF. Planejamento de experimentos e otimização de processos: uma estratégia seqüencial de planejamentos. Brasil: $1^{\text {a }}$ Ed., Editora Casa do Pão - Campinas, SP, 2009. 316 p. 